\title{
Comparison of the Clinical and Laboratory Findings and Outcomes of Hospitalized COVID-19 Patients who Were Vaccinated with Coronavac or Unvaccinated: An Analytical, Cross Sectional Study
}

\section{Serap Şimşek-Yavuz ( $\square$ serapsimsekyavuz@gmail.com )}

Istanbul University Istanbul Faculty of Medicine: Istanbul Universitesi Istanbul Tip Fakultesi https://orcid.org/0000-0002-4675-169X

\section{Gülşah Tunçer}

Haseki Training and Research Hospital: Istanbul Haseki Egitim Ve Arastirma Hastanesi

Özlem Altuntaş Aydın

Sağlık Bilimleri Üniversitesi: Saglik Bilimleri Universitesi

\section{Mehtap Aydın}

Umraniye Training and Research Hospital: Umraniye EAH

\section{Filiz Pehlivanoğlu}

Haseki Training and Research Hospital: Istanbul Haseki Egitim Ve Arastirma Hastanesi

\section{Yeşim Tok}

Cerrahpasa Medical School: Istanbul Universitesi-Cerrahpasa Cerrahpasa Tip Fakultesi

\section{Sevim Meşe}

Istanbul University Istanbul Faculty of Medicine: Istanbul Universitesi Istanbul Tip Fakultesi

Alper Gündüz

Sağlık Bilimleri Üniversitesi: Saglik Bilimleri Universitesi

\section{Ceyda Geyiktepe Güçlü}

Haseki Training and Research Hospital: Istanbul Haseki Egitim Ve Arastirma Hastanesi

İklima Özdoğan

Umraniye Training and Research Hospital: Umraniye EAH

\section{Börçe Hemiş Aydın}

Istanbul University Istanbul Faculty of Medicine: Istanbul Universitesi Istanbul Tip Fakultesi

Pınar Soğuksu

Istanbul University Istanbul Faculty of Medicine: Istanbul Universitesi Istanbul Tip Fakultesi

Aysun Benli

Istanbul University Istanbul Faculty of Medicine: Istanbul Universitesi Istanbul Tip Fakultesi

\section{Seniha Başaran}

Istanbul University Istanbul Faculty of Medicine: Istanbul Universitesi Istanbul Tip Fakultesi

Kenan Midilli 
Istanbul University Cerrahpasa Faculty of Medicine: Istanbul Universitesi-Cerrahpasa Cerrahpasa Tip Fakultesi

\section{Haluk Eraksoy}

Istanbul University Istanbul Faculty of Medicine: Istanbul Universitesi Istanbul Tip Fakultesi

\section{Research Article}

Keywords: COVID-19, Breakthrough COVID-19, inactivated SARS-CoV-2 vaccine, Coronavac

Posted Date: January 12th, 2022

DOI: https://doi.org/10.21203/rs.3.rs-1063869/v1

License: (9) This work is licensed under a Creative Commons Attribution 4.0 International License. Read Full License 


\section{Abstract}

\section{Purpose}

COVID-19 vaccines have been shown to be highly effective; however, vaccine breakthrough infections resulting from hospitalization may still occur in a small percentage of vaccinated individuals. We investigated whether the clinical and microbiological features and outcomes were different between hospitalized COVID-19 vaccinated and unvaccinated COVID-19 patients.

\section{Methods}

This multicentre, prospective, cross-sectional study was performed between April and June 2021. All hospitalized COVID-19 patients who previously had at least one dose of Coronavac were included in the study, along with some unvaccinated patients. All epidemiologic, clinical and laboratory data of the patients were recorded and compared between the vaccinated and unvaccinated individuals.

\section{Results}

There were 69 fully Coronavac vaccinated and 175 unvaccinated patients. All breakthrough infections occurred in the first 3 months of vaccination. Fully vaccinated patients were older and had more comorbidities than unvaccinated patients $(\mathrm{p}<0.05)$. There were minor differences between the groups in the symptoms, physical and laboratory findings, anti-spike IgG positivity rate, mean anti-SARS-CoV-2 S antibody level, and severity of COVID-19. The mortality rate of fully vaccinated patients was higher than the mortality rate in unvaccinated patients; however, vaccination was not an independent risk factor for mortality.

\section{Conclusions}

Hospitalized patients with breakthrough COVID-19 after Coronavac vaccination were usually older with comorbidities. The severity and clinical outcomes of these cases were similar to those of unvaccinated patients. Our findings suggest that the immune response elicited by Coronovac could be insufficient to prevent COVID-19-related severe disease and death within 3 months of vaccination among elderly people with comorbidities.

\section{Introduction}

After the administration of almost 8 billion doses of COVID-19 vaccines worldwide, it was understood that vaccines are highly effective in reducing COVID-19-related hospitalization, serious illnesses and death; however, the effectiveness of the vaccines varied by the vaccine type and sharply reduced over time; therefore, vaccine breakthrough infections resulting in hospitalization and death may occur in a small number of vaccinated, especially older and immunocompromised patients [1-4]. It was recently reported that $2.6 \%$ of fully vaccinated elderly individuals achieved a breakthrough SARS-CoV-2 infection, $20 \%$ and $2.2 \%$ of breakthrough infections needed hospitalization and resulted in death, respectively, and 
$34 \%$ of breakthrough hospitalizations needed ICU care [5]. Vaccinated people requiring hospital care due to severe COVID-19 breakthrough infection could have different clinical and laboratory features due to trained and previously acquired immunity. There are some reports of clinical and laboratory features of vaccine breakthrough infections after vaccinations with mRNA or adenoviral SARS-CoV-2 vaccines, but there is no article on the clinical characteristics of vaccine breakthrough infections after vaccination with Coronavac, which is an inactivated virus vaccine. In this study, we compared the clinical and microbiological features and outcomes of COVID-19 between Coronavac vaccinated and unvaccinated patients.

\section{Material And Methods}

This was a multicentre, prospective, analytical cross-sectional study. Patients who had at least one dose of Coronovac, had confirmed COVID-19 in the following days and were hospitalized in one of the five study hospitals between 01 April and 01 June 2021 were included in the study. Patients who had two doses of Coronavac separated at least 14 days and whose final dose was given at least 14 days earlier were accepted as fully vaccinated. For each vaccinated patient included in the study, 1 to 2 patients hospitalized in the same study hospital due to confirmed COVID-19, had no COVID-19 vaccination previously and preferably with similar comorbidities, and age was included as the control group. All epidemiologic, clinical and laboratory data of the patients were recorded in previously prepared forms. The clinical severity of COVID-19 on admission was determined with the National Early Warning Score (NEWS) [6], and the progression of all patients was evaluated and compared with the "Ordinal Scale for Clinical Improvement" of the World Health Organization (WHO) [7], which was defined on admission and on days $3,5,7,10,14$ and 21 of hospitalization. One nasopharyngeal swab and 1 plain tube of blood were taken if possible on the day of admission for the determination of SARS-CoV-2 RNA, variants of concerns (VOCs) and SARS-CoV-2 spike IgG antibody. PCR for SARS-CoV-2 RNA was performed on a Rotor-Gene Q 5 Plex Real Time PCR device (Qiagen, Germany) using a Bio-Speedy ${ }^{\circledR}$ RT-qPCR detection kit (Bioeksen R\&D Technologies Limited Company, Turkey). SARS-CoV-2 variant analysis was performed using a multiplex qRT-PCR assay (geneMAP ${ }^{T M}$ variant detection kit kindly provided by the manufacturer Genmark Sağlık Ürünleri, Istanbul, Turkey), which is designed for the detection and identification of VOCs. SARS-CoV-2 IgG antibodies were detected by ELISA using an anti-SARS-CoV-2 IgG kit (Euroimmun, Germany). The epidemiologic, clinical and laboratory features of the vaccinated and unvaccinated patients were compared. Comparisons were performed using the chi-square, independent sample t test or Mann-Whitney $U$ tests, where appropriate. Independent risk factors for mortality were defined by logistic regression analysis. IBM SPSS Statistics Version 21 was used for all of the statistical analysis.

The Ethical Committee of Istanbul University, Istanbul Faculty of Medicine approved the study on July 9, 2021 , with a decision number of 14 .

\section{Results}


A total of 292 patients who were hospitalized due to confirmed COVID-19 and either Coronavac vaccinated or not vaccinated previously were included in the study. Six patients were removed as they were vaccinated with the Biontec-mRNA COVID-19 vaccine. While $175(60.8 \%)$ of the patients were unvaccinated, 111 received at least one dose of Coronavac. Twenty-five and 86 of the patients had one and two shots of vaccination, respectively. Sixty-nine patients were accepted as fully vaccinated as they were given two shots, with the second dose for at least 14 days earlier. The mean age of all cohorts was $66.44 \pm 13.88 \mathrm{y}$, and 148 (51.4\%) were female. All breakthrough infections occurred in the first 3 months of vaccination, and the mean time from the second dose to COVID-19 was $38.42 \pm 16.82$, the median was 38 (range 14-84) days; the 25th, 50th, 75th and 100th percentiles were $25,38,50$ and 84 days, respectively.

Fully vaccinated patients were older and more frequently had sore throat, hypertension, chronic kidney disease, higher fever, higher blood pressure, higher serum creatinine and troponin levels and a higher hospital mortality rate than unvaccinated patients $(p<0.05$, Table 1$)$. 
Table 1

Comparison of the clinical and laboratory features and outcomes of patients who had either breakthrough COVID-19 after Coronavac vaccination or primary COVID-19.

\begin{tabular}{|c|c|c|c|}
\hline Characteristics or results & $\begin{array}{l}\text { Patients without any } \\
\text { SARS-CoV-2 vaccination } \\
\text { previously } \\
\text { Total number: } 217\end{array}$ & $\begin{array}{l}\text { Patients vaccinated } \\
\text { with two doses of } \\
\text { Coronavac } \\
\text { at least } 14 \text { days } \\
\text { earlier ( } \mathrm{n} \text { ) } \\
\text { Total number: } 69\end{array}$ & $p$ \\
\hline Age (y) mean $\pm S D$ & $65.57 \pm 13.95$ & $75.63 \pm 9.06$ & $<0.001$ \\
\hline Gender, female, n (\%) & $111(51.2)$ & $37(53.6)$ & 0.720 \\
\hline $\begin{array}{l}\text { Duration of symptoms, day, mean } \\
\pm S D\end{array}$ & $7.88 \pm 3.69$ & $6.95 \pm 3.40$ & 0.064 \\
\hline Symptoms & $76(35.2)$ & $17(24.6)$ & 0.104 \\
\hline Fever, n (\%) & $49(22.6)$ & $9(13)$ & 0.086 \\
\hline Shivering, $\mathrm{n}(\%)$ & $141(65)$ & $48(69.6)$ & 0.483 \\
\hline Cough, n (\%) & $44(20.3)$ & $11(15.9)$ & 0.426 \\
\hline Productive cough, $\mathrm{n}(\%)$ & $146(67.3)$ & $48(69.6)$ & 0.723 \\
\hline Fatigue, n (\%) & $173(79.7)$ & $47(68.1)$ & 0.046 \\
\hline Dyspnoea, n (\%) & $26(12)$ & $16(23.2)$ & 0.022 \\
\hline Sore throat, n (\%) & $18(8.3)$ & $3(4.3)$ & 0.274 \\
\hline Rhinorrhoea, n (\%) & $28(12.9)$ & $10(14.5)$ & 0.735 \\
\hline Anosmia, n (\%) & $29(13.4)$ & $14(20.3)$ & 0.161 \\
\hline Dysgeusia, n (\%) & $58(26.7)$ & $20(29)$ & 0.714 \\
\hline Headache, n (\%) & $105(48.4)$ & $31(44.9)$ & 0.616 \\
\hline Myalgia, arthralgia, n (\%) & $41(19)$ & $20(29)$ & 0.078 \\
\hline Nausea, vomiting, $\mathrm{n}(\%)$ & $21(9.7)$ & $6(8.7)$ & 0.808 \\
\hline Diarrhea, n (\%) & & & \\
\hline
\end{tabular}




\begin{tabular}{|c|c|c|c|}
\hline Characteristics or results & $\begin{array}{l}\text { Patients without any } \\
\text { SARS-CoV-2 vaccination } \\
\text { previously } \\
\text { Total number: } 217\end{array}$ & $\begin{array}{l}\text { Patients vaccinated } \\
\text { with two doses of } \\
\text { Coronavac } \\
\text { at least } 14 \text { days } \\
\text { earlier ( } n \text { ) } \\
\text { Total number: } 69\end{array}$ & $p$ \\
\hline Comorbidities & $94(43.3)$ & $50(72.5)$ & $<0.001$ \\
\hline Hypertension, n (\%) & $72(33.2)$ & $31(44.9)$ & 0.077 \\
\hline Diabetes Mellitus, n (\%) & $35(16.1)$ & $16(23.2)$ & 0.182 \\
\hline Chronic lung diseases, $\mathrm{n}(\%)$ & $15(6.9)$ & $12(17.4)$ & 0.010 \\
\hline Chronic kidney disease, $\mathrm{n}(\%)$ & $41(18.9)$ & $17(24.6)$ & 0.301 \\
\hline Coronary artery disease, $\mathrm{n}(\%)$ & $24(11.1)$ & $13(18.8)$ & 0.096 \\
\hline Other heart disease, $\mathrm{n}(\%)$ & $0(0)$ & $0(0)$ & - \\
\hline HIV infection, n (\%) & $3(1.4)$ & $2(2.9)$ & 0.403 \\
\hline SOT, n (\%) & $0(0)$ & $1(1.5)$ & 0.239 \\
\hline HCT, n (\%) & $17(7.8)$ & $9(13)$ & 0.190 \\
\hline Cancer, n (\%) & $15(6.9)$ & $5(7.2)$ & 0.925 \\
\hline \multicolumn{4}{|l|}{ Immunosuppressive treatment, $\mathrm{n}(\%)$} \\
\hline $\begin{array}{l}\text { NEWS score on admission, mean } \\
\pm S D\end{array}$ & $5.93 \pm 2.90$ & $5.48 \pm 2.77$ & 0.255 \\
\hline Presence of fever, n (\%) & $76(35.2)$ & $17(24.6)$ & 0.104 \\
\hline $\begin{array}{l}\text { Temperature on admission, }{ }^{\circ} \mathrm{C} \text {, mean } \\
\pm S D\end{array}$ & $36.53 \pm 0.56$ & $36.74 \pm 0.69$ & 0.013 \\
\hline $\begin{array}{l}\text { The highest temperature during the } \\
\text { course of the disease, }{ }^{0} \mathrm{C} \text {, mean } \pm \mathrm{SD}\end{array}$ & $37.04 \pm 0.67$ & $36.97 \pm 0.61$ & 0.485 \\
\hline Duration of fever, mean $\pm S D$ & $2.51 \pm 1.53$ & $1.60 \pm 0.69$ & 0.074 \\
\hline $\begin{array}{l}\text { Respiratory rate on admission, mean } \\
\pm \text { SD }\end{array}$ & $23.00 \pm 4.38$ & $22.71 \pm 3.95$ & 0.642 \\
\hline $\begin{array}{l}\text { The highest respiratory rate during } \\
\text { the disease course, mean } \pm S D\end{array}$ & $27.31 \pm 7.36$ & $26.26 \pm 5.69$ & 0.368 \\
\hline Heart rate on admission, mean $\pm S D$ & $86.54 \pm 15.61$ & $82.57 \pm 16.22$ & 0.080 \\
\hline $\begin{array}{l}\text { The highest heart rate during the } \\
\text { disease course, mean } \pm S D\end{array}$ & $103.04 \pm 19.34$ & $101.01 \pm 19.38$ & 0.300 \\
\hline
\end{tabular}


Characteristics or results
Patients without any

SARS-CoV-2 vaccination

previously

Total number: 217
Patients vaccinated

with two doses of

Coronavac

at least 14 days earlier ( $\mathrm{n}$ )

Total number: 69

$101.01 \pm 19.38$

0.370

$121.18 \pm 19.49$

$101.25 \pm 17.00$

$106.54 \pm 17.41$

0.009 disease course, mean $\pm S D$

$\mathrm{O}_{2}$ saturation on admission $\left(\mathrm{SpO}_{2}\right)$

$86.68 \pm 7.29$

$89.23 \pm 5.68$

0.908

mean $\pm S D$

The lowest $\mathrm{SpO}_{2}$ during the disease course, mean $\pm S D$

$\mathrm{PaO}_{2}$ on admission, mean $\pm \mathrm{SD}(\mathrm{n})$

$65.80 \pm 28.11(58)$

$68.11 \pm 34.14(18)$

0.746

The lowest $\mathrm{PaO}_{2}$ during the disease

$55.58 \pm 14.35(60)$

$56.10 \pm 17.86(19)$

0.899 course, mean $\pm S D(n)$

Unilateral involvement on thorax CT, 17 (7.9)

$\mathrm{n}(\%)$

Bilateral involvement on thorax CT, n 194 (90.7)

(\%)

50\% involvement on thorax CT, $\mathrm{n}(\%) \quad 138(64.5)$

$37(53.6)$

0.106

Blood WBC count on admission, mean $\pm S D$

$7852.58 \pm 3657.02$

$9998.26 \pm 11021.0$

0.189

The highest blood WBC count during the disease course, mean $\pm S D$

$13109.44 \pm 6179.54$

$13868.8 \pm 6578.23$

0.260

The lowest blood WBC count during the disease course, per microliter, mean $\pm S D$

Blood haemoglobin level on admission, $\mathrm{gr} / \mathrm{dL}$, mean $\pm \mathrm{SD}$

$6261.15 \pm 2706.30$

$6455.94 \pm 3171.59$

0.618

The lowest blood haemoglobin level $\quad 10.99 \pm 2.17$

during the disease course, $\mathrm{gr} / \mathrm{dL}$,

mean $\pm \mathrm{SD}$

Blood platelet count on admission, per microliter, mean $\pm S D$

The lowest blood platelet count during the disease course, per

microliter, mean $\pm S D$
$1.078 \pm 1.92$

0.455

$12.45 \pm 1.95$

$12.27 \pm 1.90$

0.500

$179874 \pm 123599$

$163791 \pm 112003$

0.337

$160023 \pm 115627$

$138031 \pm 114389$

0.169 
Characteristics or results
Patients without any

SARS-CoV-2 vaccination

previously

Total number: 217
Patients vaccinated with two doses of Coronavac

at least 14 days earlier ( $\mathrm{n}$ )

Total number: 69

$6185.49 \pm 3408.73$

$6826.38 \pm 4365.70$

0.521

Blood PNL
mean $\pm S D$

The highest blood PNL count during the disease course, mean $\pm S D$

Blood lymphocyte count on admission, mean $\pm S D$

$10897.58 \pm 5791.07$

$12463.8 \pm 8927.46$

0.246

$1133.845 \pm 821.75$

$1088.99 \pm 996.86$

0.680

The lowest blood lymphocyte count during disease course, mean $\pm S D$

$729.46 \pm 855.34$

$686.47 \pm 1194.41$

0.015

Serum CRP level on admission, $\mathrm{mg} / \mathrm{L}$, mean $\pm \mathrm{SD}$

$92.77 \pm 67.49$

$107.71 \pm 69.85$

0.076

The highest serum CRP level during the disease course, $\mathrm{mg} / \mathrm{L}$, mean $\pm S D$

Serum procalcitonin level on admission, $\mathrm{ng} / \mu \mathrm{L}$, mean $\pm \mathrm{SD}$

$125.28 \pm 87.96$

$143.23 \pm 108.37$

0.165

$0.43 \pm 2.36(190)$

$0.29 \pm 0.46(65)$

0.295

The highest serum procalcitonin level during the disease course, ng/

$1.06 \pm 5.23(186)$

$1.27 \pm 5.13(64)$

0.772

$\mu \mathrm{L}$, mean $\pm S D$

Serum ALT level on admission, $U / L$, mean $\pm S D$

$40.19 \pm 92.50$

$27.02 \pm 24.32$

0.099

The highest serum ALT level during the disease course, $U / L$, mean $\pm S D$

Serum AST level on admission, $U / L$, mean $\pm S D$

The highest serum AST level during the disease course, $\mathrm{U} / \mathrm{L}$, mean $\pm S D$

$197.98 \pm 1024.87$

$62.39 \pm 62.89$

0.019

$46.20 \pm 92.22$

$39.52 \pm 26.20$

0.896

$302.99 \pm 2160$

$66.76 \pm 76.42$

0.827

Serum creatinine level on admission, $\mathrm{mg} / \mathrm{dL}$, mean $\pm \mathrm{SD}$

$1.04 \pm 0.86$

$1.17 \pm 0.61$

0.021

The highest serum creatinine level during the disease course, $\mathrm{mg} / \mathrm{dL}$, mean $\pm S D$

Serum glucose level on admission, $\mathrm{mg} / \mathrm{dL}$, mean $\pm S D$
$1.25 \pm 1.12$

$1.33 \pm 0.72$

0.027

$173.84 \pm 97.14$

$151.27 \pm 52.46$

0.642 
Characteristics or results
Patients without any

SARS-CoV-2 vaccination

Total number: 217 previously

Patients vaccinated with two doses of Coronavac

at least 14 days earlier ( $\mathrm{n}$ )

Total number: 69

The highest serum glucose level during the disease course, $\mathrm{mg} / \mathrm{dL}$, mean $\pm S D$

\section{Serum ferritin level on admission,} $\mathrm{ng} / \mathrm{mL}$, mean $\pm \mathrm{SD}$

The highest serum ferritin level during the disease course, $\mathrm{ng} / \mathrm{mL}$, mean $\pm S D$

Serum D-dimer level on admission, $\mathrm{mg} / \mathrm{L}$, mean $\pm S D$

The highest serum D-dimer level during the disease course, $\mathrm{mg} / \mathrm{L}$, mean $\pm S D$

\section{Serum troponin level on admission,} $\mathrm{pg} / \mathrm{mL}$, mean $\pm S D(n)$

$271.27 \pm 128.43$

$259.10 \pm 100.33$

0.691

$690.04 \pm 762.00$

$485.42 \pm 512.46$

0.010

$1206.61 \pm 2360.71$

$1524.96 \pm 4265.98$

0.069
$21.18 \pm 45.92(198)$

$2.34 \pm 4.98$

0.321

$3.92 \pm 6.49$

$3.97 \pm 6.49$

0.358
$28.61 \pm 42.60(59) \quad<0.001$
The highest serum troponin level during the disease course, $\mathrm{pg} / \mathrm{mL}$, mean $\pm S D(n)$

Serum LDH level on admission, $\mathrm{U} / \mathrm{L}$, mean \pm SD

The highest serum LDH level during the disease course, $\mathrm{U} / \mathrm{L}$, mean $\pm S D$

Serum IL-6 level on admission, mean $\pm S D(n)$

The highest serum IL-6 level during the disease course, mean $\pm S D(n)$

Myocarditis, n (\%)

Acute kidney injury, n (\%)

$12(5.5)$

DIC, n (\%)

$\mathrm{O}_{2}$ supplement with nasal cannula, $\mathrm{n}$ $(\%)$

$\mathrm{O}_{2}$ supplement with face mask with reservoir, $\mathrm{n}(\%)$
$37.72 \pm 107.03(201)$

$396.55 \pm 219.31$

$722.34 \pm 2620.94$

$72.72 \pm 164.26(20)$

$94.15 \pm 96.51(11)$

$125.95 \pm 11.24(2)$

0.662

$76.84 \pm 254.90(61) \quad 0.083$

$404.42 \pm 515.57$

0.061

$467.36 \pm 241.56$

0.242

$32.77 \pm 50.08(6)$ 


\begin{tabular}{|c|c|c|c|}
\hline \multirow[t]{2}{*}{ Characteristics or results } & $\begin{array}{l}\text { Patients without any } \\
\text { SARS-CoV-2 vaccination } \\
\text { previously } \\
\text { Total number: } 217\end{array}$ & $\begin{array}{l}\text { Patients vaccinated } \\
\text { with two doses of } \\
\text { Coronavac } \\
\text { at least } 14 \text { days } \\
\text { earlier (n) }\end{array}$ & $p$ \\
\hline & Total number: 217 & Total number: 69 & \\
\hline $\mathrm{O}_{2}$ supplement with HFNC, $\mathrm{n}(\%)$ & $46(21.2)$ & $13(18.8)$ & 0.673 \\
\hline Non-invasive ventilation, $\mathrm{n}(\%)$ & $12(5.5)$ & $8(11.6)$ & 0.085 \\
\hline Mechanic ventilation, n (\%) & $16(7.4)$ & $9(13)$ & 0.146 \\
\hline Need for ICU support, n (\%) & $37(17.1)$ & $13(18.8)$ & 0.733 \\
\hline Need for vasopressors, n (\%) & $12(5.5)$ & $6(8.7)$ & 0.346 \\
\hline Need for ECMO support, n (\%) & 0 & 0 & - \\
\hline Need for CRRT, n (\%) & $4(1.8)$ & $2(2.9)$ & 0.594 \\
\hline $\begin{array}{l}\text { Total duration of favipiravir } \\
\text { treatment, day, mean } \pm S D\end{array}$ & $8.47 \pm 2.57$ & $8.56 \pm 2.17$ & 0.803 \\
\hline $\begin{array}{l}\text { Glucocorticoid use for COVID-19, n } \\
(\%)\end{array}$ & $198(91.2)$ & $63(91.3)$ & 0.988 \\
\hline $\begin{array}{l}\text { Total administered dose of } \\
\text { dexamethasone for COVID-19, mg, } \\
\text { mean } \pm S D\end{array}$ & $37.79 \pm 33.26$ & $42.54 \pm 37.76$ & 0.448 \\
\hline $\begin{array}{l}\text { Total administered dose of } \\
\text { prednisolone for COVID-19, mg, } \\
\text { mean } \pm \text { SD }\end{array}$ & $1012 .-9 \pm 829.71$ & $1202.80 \pm 926.97$ & 0.161 \\
\hline Anti-cytokine treatment for COVID- & $36(16.6)$ & $8(11.6)$ & 0.316 \\
\hline Tocilizumab, n (\%) & $18(8.3)$ & $2(2.9)$ & 0.126 \\
\hline Anakinra, n (\%) & $21(9.7)$ & $6(8.7)$ & 0.808 \\
\hline $\begin{array}{l}\text { Score of WHO ordinal scale on } \\
\text { admission, mean } \pm S D(n)\end{array}$ & $3.42 \pm 0.98(214)$ & $3.435 \pm 0.9774(69)$ & 0.674 \\
\hline $\begin{array}{l}\text { Score of WHO ordinal scale on day } 3 \\
\text { mean } \pm S D(n)\end{array}$ & $3.93 \pm 0.64(216)$ & $3.95 \pm 0.49(69)$ & 0.674 \\
\hline $\begin{array}{l}\text { Score of WHO ordinal scale on day } 5 \text {, } \\
\text { mean } \pm S D(n)\end{array}$ & $3.84 \pm 0.89(216)$ & $3.94 \pm 0.87(68)$ & 0.654 \\
\hline $\begin{array}{l}\text { Score of WHO ordinal scale on day } 7 \text {, } \\
\text { mean } \pm S D(n)\end{array}$ & $3.64 \pm 1.17(214)$ & $3.71 \pm 1.29(66)$ & 0.654 \\
\hline $\begin{array}{l}\text { Score of WHO ordinal scale on day } \\
10 \text {, mean } \pm S D(n)\end{array}$ & $3.17 \pm 1.52(209)$ & $3.27 \pm 1.61(65)$ & 0.734 \\
\hline
\end{tabular}




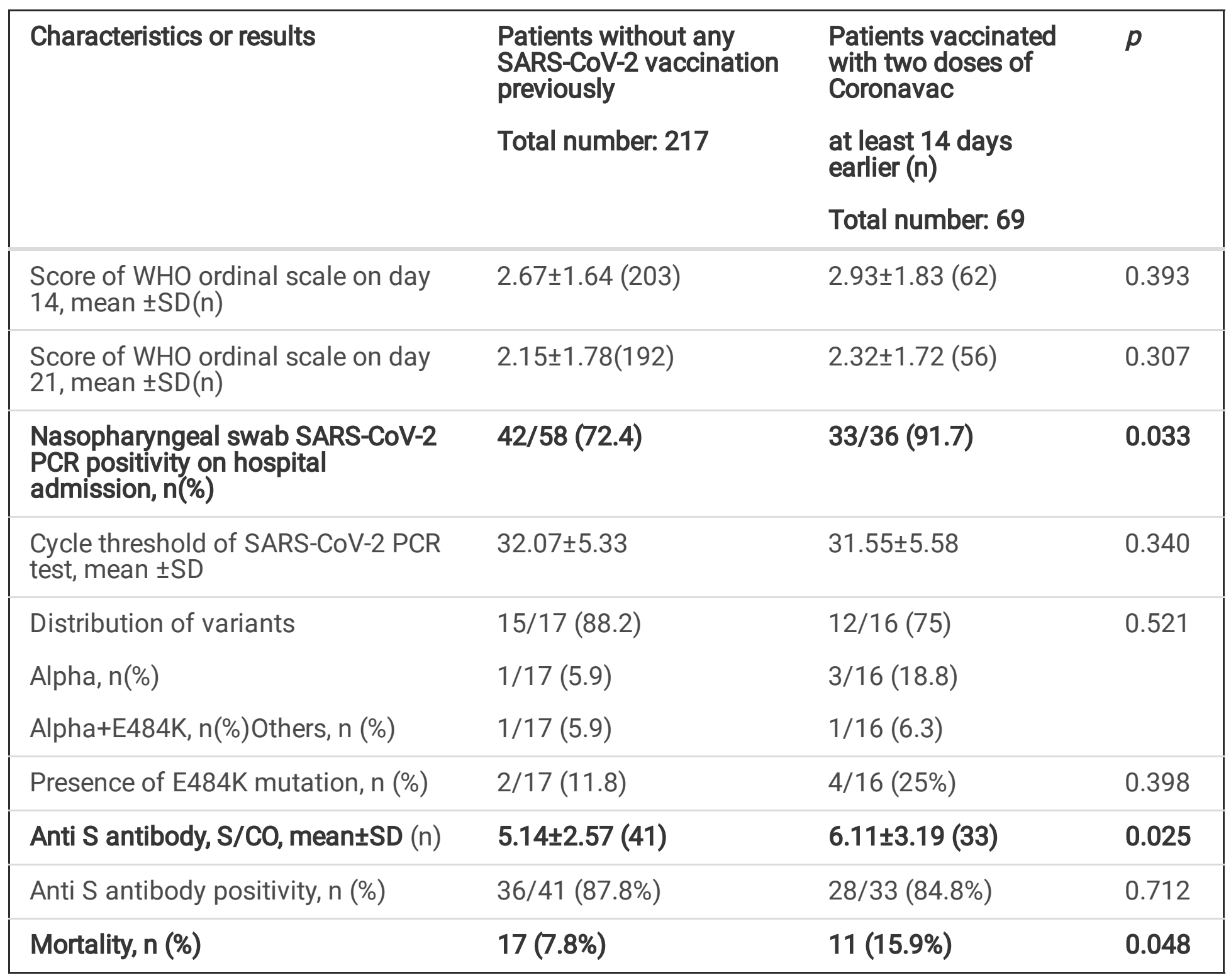

Unvaccinated patients more frequently had dyspnea, higher serum ALT and ferritin levels, and higher blood lymphocyte counts on admission $(\mathrm{p}<0.05$, Table 1$)$. NEWS scores were similar between the groups on admission and on days 3, 5, 7, 10, 14 and 21 of hospital stay. Additionally, laboratory findings on admission were similar between the vaccinated and unvaccinated patients, except vaccinated patients had higher blood WBC counts and serum troponin levels and unvaccinated patients had higher serum ALT and ferritin levels $(p<0.05)$. Results of on admission nasopharyngeal swab SARS-CoV-2 RNA PCR test and anti SARS-CoV-2 Spike IgG were available for 94 and 74 patients, respectively. The nasopharyngeal swab SARS-CoV-2 RNA PCR test positivity rate and mean anti-SARS-CoV-2 spike antibody S/CO ratio on hospital admission were higher in vaccinated patients than in unvaccinated patients $(p<0.05)$. The cycle threshold $(\mathrm{Ct})$ of the SARS-CoV-2 PCR test on hospital admission was similar between the groups. However, the Ct values were significantly higher among 46 anti SARS-CoV-2 -S antibody-positive patients than among 9 anti SARS-CoV-2 -S antibody-negative patients $(32.47 \pm 4.180$ vs $25.314 \pm 6.885)(p=0.005)$. SARS-CoV-2 variant analysis was performed for 33 patients, 16 of whom were fully vaccinated and 17 of whom were unvaccinated. The alpha variant was the most frequently isolated 
variant whose presence rate was the same in the groups. Although the alpha+E484K variant was found to be more frequent $(3 / 16(18.8 \%$ vs $1 / 17(5.9 \%))$ among patients with vaccine breakthrough infections, the difference was statistically insignificant, probably due to the small number of patients. The presence of the E484K mutation was more frequent among fully vaccinated patients (4/16 (25\%) vs $2 / 17$ (11.8)); however, the result showed no statistical significance.

In univariate analysis including all the patients, older age $(p=0.007)$, number of comorbidities $(p=0.007)$, chronic renal failure $(p=0.003)$, chronic obstructive lung disease $(p=0.009)$, heart disease other than coronary artery disease (congestive heart failure, heart valve diseases and hearth rhythm disorders) $(p=0.017)$, cancer $(p=0.017)$, full vaccination $(p=0.048)$, body temperature on admission $(p=0.011)$, hemoglobin $(p=0.021)$, procalcitonin $(p=0.001)$, creatinine $(p=0.009)$ and troponin $(p=0.001)$ levels, need for ICU ( $<<0.001)$, oxygen support with high flow nasal cannula (HFNC) or noninvasive ventilation or mechanical ventilation ( $p<0.001$, for each), development of acute renal failure due to COVID-19 $(p<0.001)$, $(p=0.009)$ were found to be risk factors for mortality.

In multivariate logistic regression analysis including all the patients, the presence of chronic lung disease, cancer, chronic renal failure and other heart diseases, the need for oxygen with HFNC, and serum hemoglobin level on admission were found to be independent risk factors for mortality (Table 2).

Table 2

Results of multivariate analysis of mortality risk factors for COVID-19.

\begin{tabular}{|c|c|c|c|c|}
\hline Variants & $\mathbf{p}$ & OR & \multicolumn{2}{|c|}{$\begin{array}{l}95 \% \text { Confidence } \\
\text { Interval (Cl) }\end{array}$} \\
\hline $\mathrm{O}_{2}$ supplement with HFNC, $\mathrm{n}(\%)$ & $<0.001$ & 6.254 & 2.230 & 17.534 \\
\hline Blood haemoglobin level on admission & 0.041 & 0.772 & 0.603 & 0.989 \\
\hline Presence of chronic lung diseases & 0.031 & 2.999 & 1.108 & 8.111 \\
\hline Presence of cancer & 0.022 & 4.345 & 1.234 & 15.296 \\
\hline Presence of chronic renal failure & 0.014 & 4.049 & 1.335 & 12.283 \\
\hline $\begin{array}{l}\text { Presence of cardiac diseases other than coronary heart } \\
\text { disease }\end{array}$ & 0.003 & 6.017 & 1.819 & 19.904 \\
\hline
\end{tabular}

The mean age of fully vaccinated patients was 75 years (ranging 53-92 years). Age, presence of chronic kidney disease, development of acute kidney injury, need for ICU care, HFNC, vasopressors and mechanical ventilation were risk factors for mortality among fully vaccinated patients. Although the $\mathrm{Ct}$ of the SARS-CoV-2 PCR test was higher for surviving patients (32.01 \pm 5.18 in 29 surviving vs $28.18 \pm 8.01$ in 4 deceased patients), the difference was statistically insignificant.

The NEWS scores on admission and on day 3 of hospital stay were not different between the deceased and surviving fully vaccinated patients, but the NEWS scores on days 5, 7, 10, 14 and 21 of hospital stay were higher in deceased patients $(p<0.05)($ Table 3$)$. 
Table 3

Comparison of the patients who recovered or died after vaccine breakthrough COVID-19.

\begin{tabular}{|c|c|c|c|c|}
\hline Characteristics or results & $\begin{array}{l}\text { Patients fully } \\
\text { vaccinated with } \\
\text { Coronavac } \\
\text { n:69 }\end{array}$ & $\begin{array}{l}\text { Fully vaccinated } \\
\text { patients who were } \\
\text { recovered after } \\
\text { COVID-19 } \\
\mathrm{n}: 58\end{array}$ & $\begin{array}{l}\text { Fully vaccinated } \\
\text { patients who have } \\
\text { died after COVID- } \\
19 \\
\mathrm{n}: 11\end{array}$ & $p$ \\
\hline Age, mean $\pm S D$ & $75.63 \pm 9.06$ & $74.53 \pm 9.18$ & $81.45 \pm 5.76$ & 0.019 \\
\hline Gender, female, n (\%) & $37(53.6)$ & $34(58.6)$ & $3(27.3)$ & 0.097 \\
\hline $\begin{array}{l}\text { Days after the second } \\
\text { dose of the vaccine, } \\
\text { mean } \pm S D\end{array}$ & $38.42 \pm 16.82$ & $33.18 \pm 14.32$ & $39.41 \pm 17.18$ & 0.263 \\
\hline Comorbidities & $50(72.4)$ & $41(70.7)$ & $9(81.8)$ & 0.715 \\
\hline Hypertension, n (\%) & $31(44.9)$ & $29(50)$ & $2(18.2)$ & 0.095 \\
\hline Diabetes Mellitus, n (\%) & $16(23.2)$ & $11(19)$ & $5(45.5)$ & 0.056 \\
\hline Chronic lung diseases, $\mathrm{n}$ & $12(17.4)$ & $7(12.1)$ & $5(45.5)$ & 0.007 \\
\hline $\begin{array}{l}\text { Chronic kidney disease, } n \\
(\%)\end{array}$ & $\begin{array}{l}17(24.6) \\
0(0)\end{array}$ & $\begin{array}{l}16(27.6) \\
0(0)\end{array}$ & $\begin{array}{l}1(9.1) \\
0(0)\end{array}$ & $\begin{array}{l}0.270 \\
-\end{array}$ \\
\hline $\begin{array}{l}\text { Coronary artery disease, } \\
\mathrm{n}(\%)\end{array}$ & $2(2.9)$ & $2(3.4)$ & $0(0)$ & 1.000 \\
\hline HIV infection, n (\%) & $\begin{array}{l}1(1.45) \\
9(13)\end{array}$ & $\begin{array}{l}1(1.7) \\
8(13.8)\end{array}$ & $\begin{array}{l}0(0) \\
1(9.1)\end{array}$ & $\begin{array}{l}1.000 \\
1.000\end{array}$ \\
\hline HCT, n (\%) & $5(7.3)$ & $5(8.6)$ & $0(0)$ & 0.585 \\
\hline $\begin{array}{l}\text { Cancer, } \mathrm{n}(\%) \\
\text { Immunosuppressive } \\
\text { treatment, } \mathrm{n}(\%)\end{array}$ & & & & \\
\hline $\begin{array}{l}\text { NEWS score on } \\
\text { admission, mean } \pm S D\end{array}$ & $5.48 \pm 2.77$ & $5.53 \pm 2.69$ & $5.18 \pm 3.28$ & 0.702 \\
\hline $\begin{array}{l}\mathrm{O}_{2} \text { saturation on } \\
\text { admission, mean } \pm \mathrm{SD}\end{array}$ & $89.23 \pm 5.68$ & $89.12 \pm 5.78$ & $89.88 \pm 5.30$ & 0.732 \\
\hline $\begin{array}{l}\text { Bilateral involvement on } \\
\text { thorax CT, } \mathrm{n}(\%)\end{array}$ & $61(88.40)$ & $52(89.7)$ & $9(81.8)$ & 0.604 \\
\hline $\begin{array}{l}>50 \% \text { involvement on } \\
\text { thorax CT, } \mathrm{n}(\%)\end{array}$ & $37(53.6)$ & $31(53.4)$ & $6(53.5)$ & 0.947 \\
\hline $\begin{array}{l}\text { Anti S S/Co ratio, mean } \\
\pm S D\end{array}$ & $6.11 \pm 3.19(33)$ & $6.53 \pm 2.99(28)$ & $3.76 \pm 3.48(5)$ & 0.034 \\
\hline
\end{tabular}




\begin{tabular}{|c|c|c|c|c|}
\hline Characteristics or results & $\begin{array}{l}\text { Patients fully } \\
\text { vaccinated with } \\
\text { Coronavac } \\
\text { n:69 }\end{array}$ & $\begin{array}{l}\text { Fully vaccinated } \\
\text { patients who were } \\
\text { recovered after } \\
\text { COVID-19 } \\
\mathrm{n}: 58\end{array}$ & $\begin{array}{l}\text { Fully vaccinated } \\
\text { patients who have } \\
\text { died after COVID- } \\
19 \\
\mathrm{n}: 11\end{array}$ & $p$ \\
\hline Myocarditis, n (\%) & $0(0)$ & 0 & 0 & - \\
\hline Acute kidney injury, $\mathrm{n}(\%)$ & $4(5.8)$ & $1(1.7)$ & $3(27.3)$ & 0.011 \\
\hline DIC, n (\%) & $0(0)$ & 0 & 0 & - \\
\hline $\begin{array}{l}\mathrm{O}_{2} \text { supplement with } \\
\text { nasal cannula, } \mathrm{n}(\%)\end{array}$ & $49(71.0)$ & $42(72.4)$ & $7(63.6)$ & 0.556 \\
\hline $\begin{array}{l}\mathrm{O}_{2} \text { supplement with } \\
\text { reservoir face mask, } \mathrm{n}(\%)\end{array}$ & $43(62.3)$ & $35(60.3)$ & $8(72.7)$ & 0.437 \\
\hline $\begin{array}{l}\mathrm{O}_{2} \text { supplement with } \\
\text { HFNC, } n(\%)\end{array}$ & $13(18.8)$ & $6(10.3)$ & $7(63.6)$ & $<0.001$ \\
\hline $\begin{array}{l}\text { Mechanic ventilation, } \mathrm{n} \\
\text { (\%) }\end{array}$ & $9(13.0)$ & $2(3.4)$ & $7(63.6)$ & $<0.001$ \\
\hline Need for ICU care, n (\%) & $13(18.8)$ & $4(6.9)$ & $9(81.8)$ & $<0.001$ \\
\hline Vasopressors, n (\%) & $6(8.7)$ & $1(1.7)$ & $5(45.5)$ & $<0.001$ \\
\hline $\begin{array}{l}\text { Total duration of } \\
\text { favipiravir treatment, day, } \\
\text { mean } \pm S D\end{array}$ & $8.56 \pm 2.17$ & $8.36 \pm 2.26$ & $9.63 \pm 1.20$ & 0.074 \\
\hline $\begin{array}{l}\text { Glucocorticoid use for } \\
\text { COVID-19, } n(\%)\end{array}$ & 63 (91.3) & 52 (89.7) & $11(100)$ & 0.580 \\
\hline $\begin{array}{l}\text { Total administered doses } \\
\text { of dexamethasone for } \\
\text { COVID- } 19, \text { mg, mean } \pm S D\end{array}$ & $42.54 \pm 37.76$ & $44.82 \pm 41.25$ & $34.80 \pm 24.14$ & 0.704 \\
\hline $\begin{array}{l}\text { Total administered doses } \\
\text { of prednisolone for } \\
\text { COVID- } 19, \text { mg, mean } \pm S D\end{array}$ & $1202.80 \pm 926.97$ & $1138.64 \pm 921.67$ & $1502.22 \pm 945.75$ & 0.290 \\
\hline Anti-cytokine treatment & $8(11.6)$ & $5(8.6)$ & $3(27.3)$ & 0.109 \\
\hline Tocilizumab, n (\%) & $\begin{array}{l}2(2.9) \\
6(8.7)\end{array}$ & $\begin{array}{l}2(3.4) \\
3(5.2)\end{array}$ & $\begin{array}{l}0(0) \\
3(27.3)\end{array}$ & $\begin{array}{l}1.000 \\
\mathbf{0 . 0 4 7}\end{array}$ \\
\hline $\begin{array}{l}\text { Score of WHO ordinal } \\
\text { scale on admission, } \\
\text { mean } \pm S D\end{array}$ & $3.46 \pm 0.90$ & $3.46 \pm 0.88$ & $3.45 \pm 1.03$ & 0.831 \\
\hline
\end{tabular}




\begin{tabular}{|c|c|c|c|c|}
\hline Characteristics or results & $\begin{array}{l}\text { Patients fully } \\
\text { vaccinated with } \\
\text { Coronavac } \\
\text { n:69 }\end{array}$ & $\begin{array}{l}\text { Fully vaccinated } \\
\text { patients who were } \\
\text { recovered after } \\
\text { COVID-19 } \\
\text { n:58 }\end{array}$ & $\begin{array}{l}\text { Fully vaccinated } \\
\text { patients who have } \\
\text { died after COVID- } \\
19 \\
\text { n: } 11\end{array}$ & $p$ \\
\hline $\begin{array}{l}\text { Score of WHO ordinal } \\
\text { scale on day } 3 \text {, mean } \\
\pm S D\end{array}$ & $3.95 \pm 0.49$ & $3.98 \pm 0.44$ & $3.81 \pm 0.75$ & 0.598 \\
\hline $\begin{array}{l}\text { Score of WHO ordinal } \\
\text { scale on day } 5 \text {, mean } \\
\pm \text { SD }\end{array}$ & $3.94 \pm 0.87$ & $3.82 \pm 0.79$ & $4.60 \pm 1.07$ & 0.029 \\
\hline $\begin{array}{l}\text { Score of WHO ordinal } \\
\text { scale on day } 7 \text {, mean } \\
\pm \text { SD }\end{array}$ & $3.71 \pm .1 .29$ & $3.44 \pm 1.15$ & $5.20 \pm 1.03$ & $<0.001$ \\
\hline $\begin{array}{l}\text { Score of WHO ordinal } \\
\text { scale on day } 10 \text {, mean } \\
\pm \text { ISD }\end{array}$ & $3.27 \pm 1.61$ & $2.90 \pm 1.40$ & $5.30 \pm 1.15$ & $<0.001$ \\
\hline $\begin{array}{l}\text { Score of WHO ordinal } \\
\text { scale on day } 14 \text {, mean } \\
\pm S D\end{array}$ & $2.93 \pm 1.83$ & $2.38 \pm 1.37$ & $5.80 \pm 1.13$ & $<0.001$ \\
\hline $\begin{array}{l}\text { Score of WHO ordinal } \\
\text { scale on day } 21 \text {, mean } \\
\pm S D\end{array}$ & $3.32 \pm 1.72$ & $1.83 \pm 1.12$ & $5.71 \pm 1.38$ & $<0.001$ \\
\hline
\end{tabular}

\section{Discussion}

In this study, we found that patients hospitalized due to breakthrough COVID-19 after Coronavac vaccination were older and had more comorbidities than unvaccinated hospitalized COVID-19 patients. The reported features of vaccine breakthrough COVID-19 cases after mRNA and/or adenovirus vector based vaccines were also quite similar the features found in our study; among cases hospitalized for COVID-19, fully vaccinated ones were older and more likely to have underlying medical conditions or to be immunosuppressed compared with unvaccinated cases [3, 8-11].

Our findings, along with others, show that older patients with comorbidities are among the most vulnerable population to severe vaccine breakthrough COVID-19. For this group of patients, close followup of vaccine response, administration of booster doses of vaccines if needed and continuation of nonpharmaceutical interventions, including social distancing and mask wearing after vaccination, are quite important.

There were minor differences in the symptoms and physical findings of COVID-19 patients who were either fully vaccinated or unvaccinated in our study. Additionally, the laboratory findings on admission of vaccinated patients were similar to the findings of unvaccinated COVID-19 patients in our cohort except 
that the mean ferritin level of vaccinated patients was lower than the levels in unvaccinated patients ( $690.04 \mathrm{ng} / \mathrm{mL}$ vs $485.42 \mathrm{ng} / \mathrm{mL}$ ). In another study from India, ferritin levels were also found to be lower in patients with vaccine breakthrough infections than in patients with primary infection $(544.82 \mathrm{ng} / \mathrm{mL}$ vs $392.26 \mathrm{ng} / \mathrm{mL}$ ) [12]; however, no significant differences were found in white blood count, absolute lymphocyte count nadir, C-reactive protein, interleukin-6, procalcitonin, oxygen saturation, lung involvement, and fever frequency between the recipients of the first and second vaccine doses in a study conducted in Poland [13]. It should be known that the clinical course or laboratory findings of patients hospitalized due to breakthrough COVID-19 after Coronavac vaccination may not reveal any distinctive features. Despite the lower ferritin level, the severity of the disease, incidence of complications including acute kidney injury, requirements for intensive care unit support and mortality rate were not different between the vaccinated and unvaccinated patients in our study; NEWS scores of vaccinated and unvaccinated patients on admission were found to be 5.53 and 5.18 , respectively. The mortality rate of fully vaccinated patients was found to be higher than the mortality rate of unvaccinated patients in univariate analysis; however, vaccination was not found to be an independent risk factor for mortality in the multivariate analysis. In contrast to our study, the severity of the disease and a requirement for ventilator support were significantly lower in the vaccinated group than in the unvaccinated patients in the Indian study mentioned above; however, the mortality rate was not found to be significantly different between the groups in that study and similar to our study, no significant differences were seen in the incidence of acute kidney injury (AKI), requirement for renal replacement therapy (RRT) or thrombotic complications between the two groups [12]. In another study of 218 mRNA-vaccinated breakthrough delta variant COVID-19 cases hospitalized in a Singapore Hospital, despite significantly older age in the vaccine breakthrough group, the odds of severe COVID-19 requiring oxygen supplementation were significantly lower following vaccination, and vaccination was found to be associated with a faster decline in viral RNA load and a robust serological response [14]. In a USA study of 67,311 laboratoryconfirmed COVID-19-associated hospitalized adult cases, there were no differences in the risk for ICU admission or in-hospital death between 30,967 unvaccinated and 1,255 fully vaccinated persons [3]. Finally, in a recent study from the USA, among patients hospitalized with COVID-19, mRNA vaccine breakthrough cases less commonly received ICU care and invasive mechanical ventilation than unvaccinated cases, and the likelihood of death was also lower in the vaccinated cases; but these findings did not differ by age group or immunocompromised status [11]. These differences in the clinical severity and outcomes of vaccinated and unvaccinated hospitalized COVID-19 patients between our study and other cohorts might be associated with two reasons: One is that compared with the Indian and Singapore cohorts [12,14], our and USA's [3] vaccinated breakthrough cases were older (mean ages were 75 and 73 vs 58 and 56 years in our and USA and Indian and Singapore studies, respectively) and had more comorbidities (prevalence of HT+ DM were $72 \%+44 \%$ and $70 \%+39 \%$ vs $51 \%$ vs $7 \%+19 \%$ in our and USA [3] and Indian and Singapore cohort [12, 14], respectively). Both older age and comorbidities are welldefined risk factors leading to severe disease and lower antibody response and protection from vaccines or higher vaccine unresponsiveness. Coronavac was found to be $42 \%$ effective among elderly people ( $>70$ years old) after $\geq 14$ days of the 2 nd dose; it declined with increasing age in a Brazilian study [15]. In another study from Poland, fully vaccinated breakthrough cases were more frequently confirmed to be 
vaccine non-responders [13]. Confirming this hypothesis, in a recent study from the UK, among 10,024 vaccinated individuals with SARS-CoV-2 infection (9479 of them were matched to unvaccinated controls), COVID-19 vaccination was found to be associated with a lower risk of several, but not all, COVID-19 sequelae in those with breakthrough SARS-CoV-2 infection, but these benefits of vaccination were clear in younger people but not in people over 60 years of age [16].

Another reason could be the type of vaccine used for vaccination. We included patients only vaccinated with Coronavac. Although all WHO-approved SARS-CoV-2 vaccines were shown to be effective against COVID-19, none of the COVID-19 vaccines created an equal level of protection, and the reported effectiveness of those vaccines against symptomatic COVID-19 varied from 50-95\% between the vaccine types. mRNA vaccines were reported to be the most effective vaccines, followed by adenoviral vaccines and then inactivated vaccines [17]. Additionally, all vaccine types, including inactivated and mRNA vaccines, were shown to have lower efficacy among elderly people in real life $[15,18]$.

It is widely accepted that protection from SARS-CoV-2 after vaccination is largely mediated by a neutralizing antibody ( $\mathrm{Nab}$ ) response, and there is a significant and positive correlation between serum anti-spike IgG levels and Nab titers $[19,20]$. Vaccine-elicited neutralization levels measured early after vaccination were found to be correlated with the subsequent protective efficacy measured in phase 3 trials [21], and the duration of immunity mainly resulted from the starting Nab levels. Because of the major differences in vaccine-elicited Nab levels between different types of vaccines, it was foreseen that the duration of protection induced by vaccines would also differ between the different vaccine types. Coronavac-elicited $\mathrm{Nab}$ titers were found to be lower than convalescent patients in a phase 2 study, and it was predicted that booster doses will be needed within 3 months after the 2 nd dose; this time span was supposed to be 9-12 months for mRNA vaccines [22]. Serum Nab titers were lower after the second dose of vaccines in both Coronavac- and Biontech mRNA-vaccinated older ( $>65$ years old) people than in younger people $[15,22]$. Additionally, the risk of symptomatic COVID-19 decreased with increasing levels of anti-spike lgG and anti-RBD IgG and Nab titer [23]. In real life, it was also seen that vaccine effectiveness decreases significantly over time. In an Israel study including almost 700.00 people, all of whom were vaccinated with the BioNTech/Pfizer mRNA BNT162b2 vaccine in a two-dose regimen, a significant correlation was found between time-from-vaccine and afforded protection against SARS-CoV2 infection [24]. BNT162b2 mRNA vaccine-induced protection against SARS-COV-2 infection was shown to wane rapidly following its peak after the second dose, but protection against hospitalization and death persisted at a robust level for 6 months after the second dose[25]. The efficacy of the Coronavac vaccine against severe delta variant infection among elderly people was shown to be 79\% 14-30 days after the second dose, and the efficacy decreased significantly 6-8 months later to $24 \%$ [26]. However, in our study, all breakthrough infections occurred during the first 3 months of vaccination, and the median time from the second dose to COVID-19 was $38.42 \pm 16.82$ days. As a result, it was concluded that not the decreasing level of Nabs but unresponsiveness or lower antibody response to the Coronavac vaccination is the main reason for the same rate of severe consequences of COVID-19 among fully vaccinated and unvaccinated cases in our cohort. As lower than $10 \%$ of our fully vaccinated cases had immunosuppressive treatment or conditions including SOT, BMT or active cancer chemotherapy in our 
cohort, the main reason for vaccine ineffectiveness was immune senescence due to older age and other comorbidities. Our finding of the same rate of SARS-CoV-2 spike antibody positivity among fully vaccinated and unvaccinated patients also supports this idea. In a study of breakthrough COVID-19 after mRNA vaccinations, the frequency of detectable anti-Spike antibodies in vaccinated individuals was found to be higher (100\%) than that in unvaccinated individuals (16\%) [14].

Although the mean S/CO of anti-S IgG on the 7th day of complaints among Coronavac-vaccinated inpatients was higher than that among unvaccinated inpatients in our study, the numbers were found to be very close to one another, at 6.11 vs 5.14 , respectively. However, it was shown that SARS-CoV-2 breakthrough infections are a strong booster of the humoral immune response among fully vaccinated patients, the anti-spike IgG antibody levels were increased significantly as low as 2 to 4 days after the onset of symptoms of breakthrough infections, and the levels were more than 10-fold higher in fully vaccinated infected individuals than in unvaccinated uninfected individuals $[12,27,28]$. These findings also support the lower response rate to the Coronavac vaccine among our cohort.

Corbett et al. recently described the immune correlates of protection by mRNA SARS-CoV-2 vaccine in a primate model. They found that S-specific IgG thresholds for protection from pneumonia and URT infections were $>336 \mathrm{IU} / \mathrm{mL}$ and $>645 \mathrm{IU} / \mathrm{mL}$, respectively [29]. Those numbers translate into $7.5 \mathrm{~S} / \mathrm{CO}$ and $14.5 \mathrm{~S} / \mathrm{CO}$ levels, respectively, in the Eurolmmune kit that was used in our study [30]. Our mean anti-S IgG levels of $5 \mathrm{~S} / \mathrm{CO}$ and $6 \mathrm{~S} / \mathrm{CO}$ among unvaccinated and vaccinated patients, respectively, were lower than the estimated level of protection for pneumonia despite the rapidly boosting effect of breakthrough infections among vaccinated people [28]. However, a protective threshold level of anti-SARS-CoV-2 spike IgG is not fully defined at the moment; as a result, it is impossible to draw a final conclusion with these results, and studies determining the protective level of antibodies against symptomatic COVID-19 and COVID-19 pneumonia are urgently needed. This type of level would be useful, especially among elderly and immunocompromised persons in whom unresponsiveness to the vaccine is expected.

We found a higher percentage of SARS-CoV-2 PCR positivity on admission among vaccinated patients, which could also be a reflection of the older age and comorbidities of those patients. We found no significant difference in Ct values between vaccinated and unvaccinated groups infected with SARS-CoV2. There were numerous studies with similar results, especially during the delta surge [31-34]. Given the substantial proportion of asymptomatic vaccine breakthrough cases with high viral loads, interventions, including masking and distancing, should be considered for all vaccinated persons especially in the settings with elevated COVID-19 transmission [35].

This study was performed during the alpha peak of the COVID-19 pandemic in Turkey, and as a result, most of our included patients were infected with the alpha variant. The E484 mutation, either with alpha mutations or by itself, was more frequently determined in vaccinated people in our study; however, it showed no statistical significance. The E484K mutation is known to reduce susceptibility to antibodymediated neutralization and is more prone to vaccine escape, which is also supported by our results [36]. 
Although Coronavac vaccination has been shown to reduce COVID-19-related hospitalization [37], our study is the first to evaluate the association between vaccination with Coronavac and progression to critical illness among hospitalized COVID-19 patients.

Our study has some limitations. As we included only hospitalized COVID-19 patients, we were unable to describe the effect of Coronavac on reducing hospitalization among COVID-19 patients. Additionally, while we included all the Coronavac vaccinated hospitalized COVID-19 cases, we were not able to include all the unvaccinated COVID-19 patients due to the high number of patients because of the COVID-19 alfa surge in Turkey during the study period. This could result in selection biases of unvaccinated patients and could affect the results of the comparisons, but the inclusion of nearly 3 times more unvaccinated patients than vaccinated patients could increase the power of our analysis.

\section{Conclusions}

Elderly people with comorbidities, who have been vaccinated with Coronavac should continue to practice non-pharmaceutical interventions, including social distancing and mask-wearing after vaccination, because of the ongoing risk of severe COVID-19, especially during uncontrolled infection transmission in the community. Our findings, including the occurrence of breakthrough COVID-19 in the first three months of vaccination, the same clinical and laboratory features and outcomes of COVID-19, the same rate of anti-spike IgG positivity, and minor differences in antibody levels among fully Coronavac vaccinated and unvaccinated patients, suggest that the immune response elicited by Coronavac could be insufficient to prevent COVID-19-related hospitalization and death within 3 months of vaccination among elderly individuals with comorbidities. It also suggests that the problem is not decreasing immunity by time but the insufficient immune response to the Coronavac among this group of patients from the beginning of the vaccination. Considering three or more doses for primary immunization with Coronavac, heterologous vaccinations with different vaccine types or using more immunogenic vaccines rather than Coronavac could be the solutions in which studies should be done.

\section{Declarations}

a. Funding: GeneMAP ${ }^{\mathrm{TM}}$ variant detection kit kindly provided by the manufacturer Genmark Sağlık Ürünleri, Istanbul, Turkey. Other expenses were supported by authors.

b. Conflict of interests: None

c. Acknowledgement: None

d. Avalibility of data: All the data and consent forms are available and could be obtained from the corresponding author.

e. IBM SPSS Statistics Version 21 was obtained via Istanbul University Faculty of Medicine a registered user of the programme.

f. The Ethical Committee of Istanbul University, Istanbul Faculty of Medicine approved the study on July 9, 2021, with a decision number of 14 . 
g. Author's contributions: Serap Şimşek-Yavuz: Designed the study, analysed the data, wrote the paper, Gülşah Tuncer: Collected the data, Özlem Altunaş Aydın: Collected the data, Mehtap Aydın: Collected the data, Filiz Pehlivanoğlu: Collected the data, Yeşim Tok: Performed analysis, Sevim Meşe: Performed analysis, Alper Gündüz: Collected the data , Ceyda Geyiktepe Güçlü: Collected the data, İklima Özdoğan: Collected the data, Börçe Hemiş: Collected the data, Pınar Soğuksu: Performed analysis, Aysun Benli: Collected the data, Seniha Başaran: Collected the data, Kenan Midilli: Performed analysis, Haluk Eraksoy: Collected the data.

h. Freely-given, informed consent to participate in the study was taken from all of the participants of the study. The Informed Consent Form was prepared in accordance with WHO requirements.

i. Consent for publication (include appropriate statements): We, all the authors, give our consent for all of our article details to be published in the European Journal of Clinical Microbiology and Infectious Disease. Kind regards.

\section{References}

1. Hagan LM, McCormick DW, Lee C, Sleweon S, Nicolae L, Dixon T, Banta R, Ogle I, Young C, Dusseau C, Salmonson S, Ogden C, Godwin E, Ballom T, Ross T, Browne H, Harcourt JL, Tamin A, Thornburg NJ, Kirking HL, Salvatore PP, Tate JE. Outbreak of SARS-CoV-2 B.1.617.2 (Delta) Variant Infections Among Incarcerated Persons in a Federal Prison - Texas, July-August 2021. MMWR Morb Mortal Wkly Rep. 2021 Sep 24;70(38):1349-1354. doi: 10.15585/mmwr.mm7038e3. PMID: 34555009; PMCID: PMC8459894.

2. Vignier N, Bérot V, Bonnave N, Peugny S, Ballet M, Jacoud E, Michaud C, Gaillet M, Djossou F, Blanchet D, Lavergne A, Demar M, Nacher M, Rousset D, Epelboin L. Breakthrough Infections of SARS-CoV-2 Gamma Variant in Fully Vaccinated Gold Miners, French Guiana, 2021. Emerg Infect Dis. 2021 Oct;27(10):2673-2676. doi: 10.3201/eid2710.211427. Epub 2021 Jul 21. PMID: 34289335; PMCID: PMC8462339.

3. Havers FP, Pham H, Taylor CA, Whitaker M, Patel K, Anglin O, et al. COVID-19-associated hospitalizations among vaccinated and unvaccinated adults $\geq 18$ years - COVID2 NET, 13 states, January 1 - July 24, 2021. medRxiv preprint 2021; doi: https://doi.org/10.1101/2021.08.27.21262356.

4. Bahl A, Johnson S, Maine G, Garcia MH, Nimmagadda S, Qu L, Chen NW. Vaccination reduces need for emergency care in breakthrough COVID-19 infections: A multicenter cohort study. Lancet Reg Health Am. 2021 Sep 9:100065. doi: 10.1016/j.lana.2021.100065. Epub ahead of print. PMID: 34522911; PMCID: PMC8428472.

5. Thompson MG, Burgess JL, Naleway AL, Tyner H, Yoon SK, Meece J, Olsho LEW, Caban-Martinez AJ, Fowlkes AL, Lutrick K, Groom HC, Dunnigan K, Odean MJ, Hegmann K, Stefanski E, Edwards LJ, Schaefer-Solle N, Grant L, Ellingson K, Kuntz JL, Zunie T, Thiese MS, Ivacic L, Wesley MG, Mayo Lamberte J, Sun X, Smith ME, Phillips AL, Groover KD, Yoo YM, Gerald J, Brown RT, Herring MK, Joseph G, Beitel S, Morrill TC, Mak J, Rivers P, Poe BP, Lynch B, Zhou Y, Zhang J, Kelleher A, Li Y, 
Dickerson M, Hanson E, Guenther K, Tong S, Bateman A, Reisdorf E, Barnes J, Azziz-Baumgartner E, Hunt DR, Arvay ML, Kutty P, Fry AM, Gaglani M. Prevention and Attenuation of Covid-19 with the BNT162b2 and mRNA-1273 Vaccines. N Engl J Med. 2021 Jul 22;385(4):320-329. doi:

10.1056/NEJMoa2107058. Epub 2021 Jun 30. PMID: 34192428; PMCID: PMC8262622.

6. Royal College of Physicians (2021). National Early Warning Score (NEWS)

2.https://www.rcplondon.ac.uk/projects/outputs/national-early-warning-score-news-2. Accessed 01 November 2021

7. World Health Organization (2020). WHO R\&D Blueprint novel Coronavirus COVID-19 Therapeutic Trial Synopsis. https://www.who.int/blueprint/priority-diseases/key-action/COVID-

19_Treatment_Trial_Design_Master_Protocol_synopsis_Final_18022020.pdf. Accessed 01 November 2021

8. Brosh-Nissimov T, Orenbuch-Harroch E, Chowers M, Elbaz M, Nesher L, Stein M, Maor Y, Cohen R, Hussein K, Weinberger M, Zimhony O, Chazan B, Najjar R, Zayyad H, Rahav G, Wiener-Well Y. BNT162b2 vaccine breakthrough: clinical characteristics of 152 fully vaccinated hospitalized COVID19 patients in Israel. Clin Microbiol Infect. 2021 Jul 7;27(11):1652-7. doi:

10.1016/j.cmi.2021.06.036. Epub ahead of print. PMID: 34245907; PMCID: PMC8261136.

9. Cook C, Patel NJ, D'Silva KM, Hsu TY, Dilorio M, Prisco L, Martin LW, Vanni K, Zaccardelli A, Todd D, Sparks JA, Wallace ZS. Clinical characteristics and outcomes of COVID-19 breakthrough infections among vaccinated patients with systemic autoimmune rheumatic diseases. Ann Rheum Dis. 2021 Sep 6:annrheumdis-2021-221326. doi: 10.1136/annrheumdis-2021-221326. Epub ahead of print. PMID: 34489304.

10. Agrawal U, Katikireddi SV, McCowan C, Mulholland RH, Azcoaga-Lorenzo A, Amele S, Fagbamigbe AF, Vasileiou E, Grange Z, Shi T, Kerr S, Moore E, Murray JLK, Shah SA, Ritchie L, O'Reilly D, Stock SJ, Beggs J, Chuter A, Torabi F, Akbari A, Bedston S, McMenamin J, Wood R, Tang RSM, de Lusignan S, Hobbs FDR, Woolhouse M, Simpson CR, Robertson C, Sheikh A. COVID-19 hospital admissions and deaths after BNT162b2 and ChAdOx1 nCoV-19 vaccinations in 2.57 million people in Scotland (EAVE II): a prospective cohort study. Lancet Respir Med. 2021 Sep 29:S2213-2600(21)00380-5. doi: 10.1016/S2213-2600(21)00380-5. Epub ahead of print. PMID: 34599903; PMCID: PMC8480963.

11. Tenforde MW, Self WH, Adams K, Gaglani M, Ginde AA, McNeal T, Ghamande S, Douin DJ, Talbot HK, Casey JD, Mohr NM, Zepeski A, Shapiro NI, Gibbs KW, Files DC, Hager DN, Shehu A, Prekker ME, Erickson HL, Exline MC, Gong MN, Mohamed A, Henning DJ, Steingrub JS, Peltan ID, Brown SM, Martin ET, Monto AS, Khan A, Hough CL, Busse LW, Ten Lohuis CC, Duggal A, Wilson JG, Gordon AJ, Qadir N, Chang SY, Mallow C, Rivas C, Babcock HM, Kwon JH, Halasa N, Chappell JD, Lauring AS, Grijalva CG, Rice TW, Jones ID, Stubblefield WB, Baughman A, Womack KN, Rhoads JP, Lindsell CJ, Hart KW, Zhu Y, Olson SM, Kobayashi M, Verani JR, Patel MM; Influenza and Other Viruses in the Acutely III (IVY) Network. Association Between mRNA Vaccination and COVID-19 Hospitalization and Disease Severity. JAMA. 2021 Nov 4. doi: 10.1001/jama.2021.19499. Epub ahead of print. PMID: 34734975. 
12. Jagadeesh Kumar V, Sowpati DT, Munigela A, Banu S, Siva AB, Sasikala M, et al. Clinical outcomes in vaccinated individuals hospitalized with Delta variant of SARS-CoV-2. medRxiv preprint2021; doi:https://doi.org/10.1101/2021.07.13.21260417.

13. Rzymski P, Pazgan-Simon M, Simon K, Łapiński T, Zarębska-Michaluk D, Szczepańska B, Chojnicki M, Mozer-Lisewska I, Flisiak R. Clinical Characteristics of Hospitalized COVID-19 Patients Who Received at Least One Dose of COVID-19 Vaccine. Vaccines (Basel). 2021 Jul 13;9(7):781. doi: 10.3390/vaccines9070781. PMID: 34358197.

14. Chia PY, Ong SWX, Chiew CJ, Ang LW, Jean-Chavatte M, Mak TM, et al. Virological and serological kinetics of 1 SARS-CoV-2 Delta variant vaccine breakthrough infections: a multi-center cohort study. medRxiv preprint 2021; doi: https://doi.org/10.1101/2021.07.28.21261295

15. Ranzani OT, Hitchings M, Nieto MD, D’Agostini TL, Paula RC, Oaula OFPi et al. Effectiveness of the CoronaVac vaccine in the elderly population during a P.1 variant-associated epidemic of COVID-19 in Brazil: A test-negative case-control study, medRxiv preprint 2021; doi: https://doi.org/10.1101/2021.05.19.21257472;

16. Taquet M, Dercon Q, Harrison PJ. Six-month sequelae of post-vaccination SARS-CoV-2 infection: a retrospective cohort study of 10,024 breakthrough infections. medRxiv preprint 2021; doi: https://doi.org/10.1101/2021.10.26.21265508.

17. Wadman M. The overlooked superpower of mRNA vaccines. Science. 2021 Jul 30;373(6554):479. doi: 10.1126/science.373.6554.479. PMID: 34326217.

18. Moustsen-Helms IR, Emborg HD, Nielsen J, Nielsen KF, TG Krause, Mølbak K, et al. Vaccine effectiveness after 1st and 2nd dose of the BNT162b2 mRNA Covid-19 Vaccine in long-term care facility residents and healthcare workers - a Danish cohort study. medRxiv preprint 2021; doi: https://doi.org/10.1101/2021.03.08.21252200

19. Israelow B, Mao T, Klein J, Song E, Menasche B, Omer SB, Iwasaki A. Adaptive immune determinants of viral clearance and protection in mouse models of SARS-CoV-2. Sci Immunol. 2021 Oct 15;6(64):eabl4509. doi: 10.1126/sciimmunol.abl4509. Epub 2021 Sep 2. PMID: 34623900.

20. Liu J, Bodnar BH, Wang X, et al. Correlation of vaccine-elicited antibody levels and neutralizing activities against SARS-CoV-2 and its variants. bioRxiv 2021; doi:10.1101/2021.05.31.445871.

21. Khoury DS, Cromer D, Reynaldi A, Schlub TE, Wheatley AK, Juno JA, Subbarao K, Kent SJ, Triccas JA, Davenport MP. Neutralizing antibody levels are highly predictive of immune protection from symptomatic SARS-CoV-2 infection. Nat Med. 2021 Jul;27(7):1205-1211. doi: 10.1038/s41591-02101377-8. Epub 2021 May 17. PMID: 34002089.

22. Levin EG, Lustig Y, Cohen C, Fluss R, Indenbaum V, Amit S, Doolman R, Asraf K, Mendelson E, Ziv A, Rubin C, Freedman L, Kreiss Y, Regev-Yochay G. Waning Immune Humoral Response to BNT162b2 Covid-19 Vaccine over 6 Months. N Engl J Med. 2021 Oct 6. doi: 10.1056/NEJMoa2114583. Epub ahead of print. PMID: 34614326.

23. Feng S, Phillips DJ, White T, Sayal H, Aley PK, Bibi S, Dold C, Fuskova M, Gilbert SC, Hirsch I, Humphries HE, Jepson B, Kelly EJ, Plested E, Shoemaker K, Thomas KM, Vekemans J, Villafana TL, 
Lambe T, Pollard AJ, Voysey M; Oxford COVID Vaccine Trial Group. Correlates of protection against symptomatic and asymptomatic SARS-CoV-2 infection. Nat Med. 2021 Sep 29. doi:

10.1038/s41591-021-01540-1. Epub ahead of print. PMID: 34588689.

24. Mizrahi B, Lotan R, Kalkstein N, Peretz A, Perez G, Ben-Tov A, et al. Correlation of SARS-CoV-2 Breakthrough Infections to Time-from-vaccine; Preliminary Study. medRxiv preprint 2021; doi: https://doi.org/10.1101/2021.07.29.21261317

25. Chemaitelly H, Tang P, Hasan MR, AlMukdad S, Yassine HM, Benslimane FM, Al Khatib HA, Coyle P, Ayoub HH, Al Kanaani Z, Al Kuwari E, Jeremijenko A, Kaleeckal AH, Latif AN, Shaik RM, Abdul Rahim HF, Nasrallah GK, Al Kuwari MG, Al Romaihi HE, Butt AA, Al-Thani MH, Al Khal A, Bertollini R, AbuRaddad LJ. Waning of BNT162b2 Vaccine Protection against SARS-CoV-2 Infection in Qatar. N Engl J Med. 2021 Oct 6. doi: 10.1056/NEJMoa2114114. Epub ahead of print. PMID: 34614327.

26. Chen X, Wang W, Chen X, Wu Q, Sun R, Ge S, et al. Prediction of long-term kinetics of vaccineelicitedneutralizing antibody and time-varying vaccine-specific efficacy against the SARS-CoV-2 Delta variant by clinical endpoint. medRxiv preprint 2021; doi:

https://doi.org/10.1101/2021.09.23.21263715.

27. Müller L, Andrée M, Ostermann PN, Jazmati N, Flüh G, Fischer J, et al.SARS-CoV-2 infection in fully vaccinated individuals of old age strongly boosters the humoral immune response. medRxiv preprint 2021; doi: https://doi.org/10.1101/2021.07.19.21260563

28. Hacisuleyman E, Hale C, Saito Y, Blachere NE, Bergh M, Conlon EG, Schaefer-Babajew DJ, DaSilva J, Muecksch F, Gaebler C, Lifton R, Nussenzweig MC, Hatziioannou T, Bieniasz PD, Darnell RB. Vaccine Breakthrough Infections with SARS-CoV-2 Variants. N Engl J Med. 2021 Jun 10;384(23):2212-2218. doi: 10.1056/NEJMoa2105000. Epub 2021 Apr 21. PMID: $33882219 ;$ PMCID: PMC8117968.

29. Corbett KS, Nason MC, Flach B, Gagne M, O'Connell S, Johnston TS, Shah SN, Edara VV, Floyd K, Lai L, McDanal C, Francica JR, Flynn B, Wu K, Choi A, Koch M, Abiona OM, Werner AP, Moliva JI, Andrew SF, Donaldson MM, Fintzi J, Flebbe DR, Lamb E, Noe AT, Nurmukhambetova ST, Provost SJ, Cook A, Dodson A, Faudree A, Greenhouse J, Kar S, Pessaint L, Porto M, Steingrebe K, Valentin D, Zouantcha S, Bock KW, Minai M, Nagata BM, van de Wetering R, Boyoglu-Barnum S, Leung K, Shi W, Yang ES, Zhang Y, Todd JM, Wang L, Alvarado GS, Andersen H, Foulds KE, Edwards DK, Mascola JR, Moore IN, Lewis MG, Carfi A, Montefiori D, Suthar MS, McDermott A, Roederer M, Sullivan NJ, Douek DC, Graham BS, Seder RA. Immune correlates of protection by mRNA-1273 vaccine against SARS-CoV-2 in nonhuman primates. Science. 2021 Sep 17;373(6561):eabj0299. doi: 10.1126/science.abj0299. Epub 2021 Sep 17. PMID: 34529476; PMCID: PMC8449013.

30. Bradley BT, Bryan A, Fink SL, Goecker EA, Roychoudhury P, Huang ML,et al. Anti-SARS-CoV-2 Antibody Levels Measured by the AdviseDx SARS-CoV-2 Assay Are Concordant with Previously Available Serologic Assays but Are Not Fully Predictive of Sterilizing Immunity. J Clin Microbiol. 2021 Aug 18;59(9):e0098921. doi: 10.1128/JCM.00989-21. Epub 2021 Aug 18. PMID: 34165323; PMCID: PMC8373027

31. Ramirez E, Wilkes RP, Carpi G, Dorman J, Bowen C, Smith L. SARS-CoV-2 Breakthrough Infections in Fully Vaccinated Individuals. medRxiv preprint 2021; doi: 
https://doi.org/10.1101/2021.06.21.21258990.

32. Shamier MC, Tostmann A, Bogers S, Wilde J, IJpelaar J, Kleij WA, et al. Virological characteristics of SARS-CoV-2 vaccine breakthrough infections in health care workers. medRxiv preprint 2021; doi: https://doi.org/10.1101/2021.08.20.21262158.

33. Amit S, Gonen T, Regev-Yochay G. Covid-19 Breakthrough Infections in Vaccinated Health Care Workers. Reply. N Engl J Med. 2021 Oct 21;385(17):1630-1631. doi: 10.1056/NEJMc2113497. Epub 2021 Sep 29. PMID: 34587379.

34. Riemersma KK, Grogan BE, Kita-Yarbro A, Jeppson GE, O'Connor DH, Friedrich TC, et al. Vaccinated and unvaccinated individuals have similar viral loads in communities with a high prevalence of the SARS-CoV-2 delta variant. medRxiv preprint 2021; doi:

https://doi.org/10.1101/2021.07.31.21261387.

35. Acharya CB, Schrom J, Mitchell AM, Coil DA, Marquez C, Rojas S, et al. No Significant Difference in Viral Load Between Vaccinated and Unvaccinated, Asymptomatic and Symptomatic Groups Infected with SARS-CoV-2 Delta Variant. medRxiv preprint 2021; doi:

https://doi.org/10.1101/2021.09.28.21264262

36. Arora P, Rocha C, Kempf A, Nehlmeier I, Graichen L, Winkler MS, Lier M, Schulz S, Jäck HM, Cossmann A, Stankov MV, Behrens GMN, Pöhlmann S, Hoffmann M. The spike protein of SARS-CoV2 variant $A .30$ is heavily mutated and evades vaccine-induced antibodies with high efficiency. Cell Mol Immunol. 2021 Oct 25. doi: 10.1038/s41423-021-00779-5. Epub ahead of print. PMID: 34697413.

37. Jara A, Undurraga EA, González C, Paredes F, Fontecilla T, Jara G, Pizarro A, Acevedo J, Leo K, Leon F, Sans C, Leighton P, Suárez P, García-Escorza H, Araos R. Effectiveness of an Inactivated SARS-CoV-2 Vaccine in Chile. N Engl J Med. 2021 Sep 2;385(10):875-884. doi: 10.1056/NEJMoa2107715. Epub 2021 Jul 7. PMID: 34233097; PMCID: PMC8279092.

38. Wilder-Smith A, Mulholland K. Effectiveness of an Inactivated SARS-CoV-2 Vaccine. N Engl J Med. 2021 Sep 2;385(10):946-948. doi: 10.1056/NEJMe2111165. PMID: 34469651; PMCID: PMC8427532. 